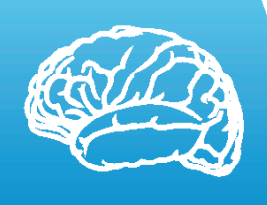

NELROLOGY
1) Department of Neurosciences, Iuliu Hatieganu Univesrity of Medicine and Pharmacy, Cluj-Napoca, Romania

2) Department of Surgery, Emergency Medicine Division, Iuliu Hatieganu Univesrity of Medicine and Pharmacy, Cluj-Napoca, Romania

3) Department of Medical Education, Medical Informatics and Biostatistics, Iuliu Hatieganu University of Medicine and Pharmacy, Cluj-Napoca, Romania

DOI: $10.15386 / \mathrm{mpr}-1783$

Manuscript received: 25.08 .2020

Received in revised form: 08.02.2021

Accepted: 01.04.2021

Address for correspondence:

adela.golea@umfcluj.ro

This work is licensed under a Creative Commons Attribution-NonCommercialNoDerivatives 4.0 International License

\section{Feasibility of a pilot study on point-of-care biomarkers in spontaneous intracerebral hemorrhage in an emergency setting}

Eugenia-Maria Mureșan ${ }^{1}$, Adela Golea ${ }^{2}$, Sorana D. Bolboacă ${ }^{3}$, Lăcrămioara Perju-Dumbravă ${ }^{1}$

\begin{abstract}
Background and aims. Stroke is a worldwide leading cause of death and disability and spontaneous intracerebral hemorrhage ( $\mathrm{sICH}$ ) has significant economic and social impact, regardless of recent efforts towards outcome-bettering acute interventions. The aim of the study was to assess the feasibility of a prospective observational research regarding point-of-care (POC) biomarkers in $\mathrm{sICH}$, conducted in a level one emergency department (ED).

Methods. Patients with acute ( $<8$ hours) sICH were enrolled in this study. Patients presenting a Glasgow Coma Scale score $<8$, secondary causes of intracerebral hemorrhage, seizures, recent ischemic events, known thromboembolic disease, anticoagulant treatment, severe pre-stroke disability, terminal disease, scheduled neurosurgery/hemostatic treatment were excluded. Feasibility was defined as ED inclusion and follow-up rates, time-to-inclusion, and frequency of missing data. Baseline demographic, imaging and POC biochemical status of the study group were documented, including inflammatory (complete blood count, C-reactive protein), metabolic (glucose, hepatic, and renal function) and cardiovascular markers (cardiac troponin I, D-dimer).
\end{abstract}

Results. The inclusion rate was 2.16 patients/month with a final sample of 35 patients out of 239 potentially eligible patients. The median time from symptom onset to ED presentation was 128 minutes (IQR 96-239), with 21/35 patients having presented within the first 3 hours from ictus. Median times between symptoms' onset to Computer Tomography (CT) scan and ED presentation to CT scan were 170 minutes (IQR 126-317) and 25 minutes (IQR 17-62), respectively. The median time from patient's presentation to CBC result was 12 minutes (IQR 6.5-20), with 21/35 study participants having the results available within 15 minutes from ED arrival. The median cohort age was 72-years, with a 19/16 male/female ratio. Hypertension was the most frequent risk factor (77\%), along with ischemic heart disease (31\%) and diabetes $(29 \%)$. One-third of the hypertensive patients did not undergo blood pressure lowering treatment. Median values of POC biomarkers on ED admission were within normal range.

Conclusions. It was feasible to determine point-of-care biomarkers in spontaneous intracerebral hemorrhage on admission in ED, despite the urgency of the medical condition.

Keywords: cerebral hemorrhage, point-of-care systems, emergency service, biomarkers, feasibility study 


\section{Background and aims}

Emergency medicine (EM) is considered a developing medical specialty, having its beginnings in the 1960s in the U.S.A., Canada, and the United Kingdom [1]. Later, the need for standardized emergency care led to its recognition as an independent specialty and the worldwide formation of professional societies. Romania is amongst the European countries where the EM specialty is officially recognized and celebrates 28 years of existence in 2021. Emergency physicians are providing $24 / 7$ care in advanced and intermediate care hospitals, addressing not only life-threatening conditions, but also stable patients in need of urgent care.

Among life-threatening conditions, stroke (ischemic or hemorrhagic) has been the focus of continual advancement, particularly considering the benefit of thrombolysis for the ischemic one. Regarding hemorrhagic stroke and intracerebral hemorrhage (ICH) in particular, definitive therapies are still being sought in the hope of bettering functional outcome [2,3]. Though responsible for only $10-20 \%$ of total stroke cases [4], ICH is considered a devastating condition not only in terms of the patient's direct impairment but also as an economic and social burden [5]. Research efforts have been focused during the last decade on identifying biomarkers that could lead to functional outcome improvement of hemorrhagic stroke patients, along with risk stratification and prognosis.

Inflammatory status on admission was one of the contributing factors to an unfavorable outcome in $\mathrm{sICH}$ patients [6], as stroke is generally affecting systems such as cardiac, pulmonary, and immune system [7]. C-reactive protein (CRP), an acute phase inflammatory marker, has been associated with increased mortality [8-10], poor outcome [9] or hematoma volume [10] and growth [11]. D-dimer, a product of fibrin degradation, proved to be a risk factor for mortality $[8,12,13]$ and early neurological deterioration (END) [14] and was associated with parenchymal hematoma volume $[12,13]$. Reports on cardiac troponin elevations (with subtypes I and $\mathrm{T}$ considered as largely offering identical information [15] are still controversially linked with mortality in spontaneous $\mathrm{ICH}(\mathrm{sICH})[9,16,17]$, yet have been shown as an indicator of poor outcome in subarachnoid hemorrhage [15].

If considering the nature of the emergency department (ED), with personnel regularly attending to new patients in need of diagnosis and immediate care, implementing prospective research protocols is a tremendous challenge even for the most experienced physicians. In Romania, only 20 prospective research protocols have been published as having recruited patients directly from ED. The PubMed search performed on May $22^{\text {nd }}, 2020$ (search strategy "(emergency department) AND (prospective) AND (Romania)" over 10 years period) retrieved 15 valid observational studies (two hundred and ninety-one titles and abstracts have been screened). Five additional articles have been identified on previous similar searches.

The 20 studies were conducted in 9 hospitals from 5 teaching medical centers and an additional nonteaching hospital. Nine articles reported results as part of international research [18-26]. Research topics included resuscitation [21,26-30], cardiovascular and respiratory pathologies (syncope [31,32], acute heart failure [22], asthma [25], and dyspnoea [24,33], infectious diseases $[19,20]$, with 1 regarding the paediatric population [19], pre-hospital [18,23], toxicology [34-36], trauma [37], and upper gastrointestinal bleeding [38,39].

Our ED has implemented one research protocol $[29,30]$ and has been part of another international research group $[24,25]$.

Bearing in mind the above, the input of point-ofcare biomarkers (POC) in risk stratification and outcome prognosis of patients presenting with sICH should be assessed throughout ED-based research.

The primary objective of the present study was to evaluate the feasibility of a prospective ED research protocol by assessing inclusion and follow-up rates, alongside time-to-inclusion and frequency of missing data. The POC evaluation of the cohort's biochemical status was the secondary objective of our study.

\section{Methods \\ Study design and setting}

Emergency Management of Spontaneous Intracerebral Hemorrhage - Biomarkers (EsICH-bio, registered at ClinicalTrials.gov - NCT02935985) was a prospective observational study that recruited $\mathrm{sICH}$ patients presenting to the ED of County Emergency Hospital Cluj-Napoca, Romania over a time frame of 18 months (12.12.2016 - 11.06.2018). The medical facility is a 1,542 beds teaching hospital in the NorthWest of Romania, providing medical care for $3.46 \%$ of the country's population [40]. The EsICH-bio study protocol was approved by the Ethics Committee of "Iuliu Haţieganu" University of Medicine and Pharmacy of Cluj-Napoca, Romania, (441/24.11.2016) and endorsed by the Ethics Committee of the hospital. The study procedures and interventions are following the principles stated by the Declaration of Helsinki.

Patients with an imaging diagnosis of acute sICH (defined as hyperdense parenchymal hemorrhagic collection of no detectable secondary cause) were 
recruited from the ED, based on a phone alert system. Informed consent was obtained from patient/proxy (or the emergency procedure (EP) form signed by an investigator and a physician not related to the study team) prior to participant's clinical assessment (including mRS - modified Rankin Scale and NIHSS - National Institute of Health Stroke Scale). A team of EM physicians (7 out of 63 attendings and residents) who received prior training (including mRS and NIHSS) completed the enrolment of the eligible patients and performed the clinical evaluation. The participation of the EM physicians was on a voluntary basis, with no financial compensations available.

Venous blood samples for the study of POC biomarkers were collected following clinical assessment, including cardiac troponin subtype I (cTnI), high-sensitive CRP (hs-CRP) and D-dimer. POC blood measurements were performed for all participants as part of the primary ED assessment and consisted of complete blood count (CBC): white blood cells (WBC), granulocytes (GRA), lymphocytes (LYM), mid-cell fractions (MID) and platelets (PLT), glucose, hepatic, and renal function. Data collection also included medical and medication history (containing cardiovascular risk factors such as age, gender, history of hypertension, diabetes mellitus, cigarette smoking, hyperlipidemia, symptomatic ischemic heart disease, and previous ischemic/ hemorrhagic stroke events), pre-treatment vital signs, medical management variables, surgical/ intensive care unit interventions, and medical complications.

The enrolment and follow-up processes are presented in table I. After ED enrolment, participants were clinically assessed on days 2 and 7 (or discharge) on neurology/ neurosurgery wards by members of the recruiting team. Telephone surveys were conducted on days 90 and 180, collecting data on functional outcome (mRS), disability (Barthel Index), quality of life (EuroQoL - Quality of Life), cognition (Telephone Interview for Cognitive Status-modified) and mood (Zung Self-Rating Depression Scale).

Feasibility criteria comprised inclusion and followup rates (on days 90 and 180), onset/ ED admission to inclusion timeframes (reported as symptom onset/ ED admission landmark to $\mathrm{CT}$ scan acquisition/ imaging diagnosis, routine blood panel (defined as time of the $\mathrm{CBC}$ results) and sampling of study specific biomarkers) and frequency of missing data. Secondary objective was the POC evaluation of the baseline biochemical status of the study cohort.

Additionally, a retrospective data collection was performed regarding patients with non-traumatic ICH who were not included in EsICH-bio, with the aim of documenting potentially eligible patients lost due to no phone alert of the research team.

Table I. EsICH-bio patient enrolment and follow-up.

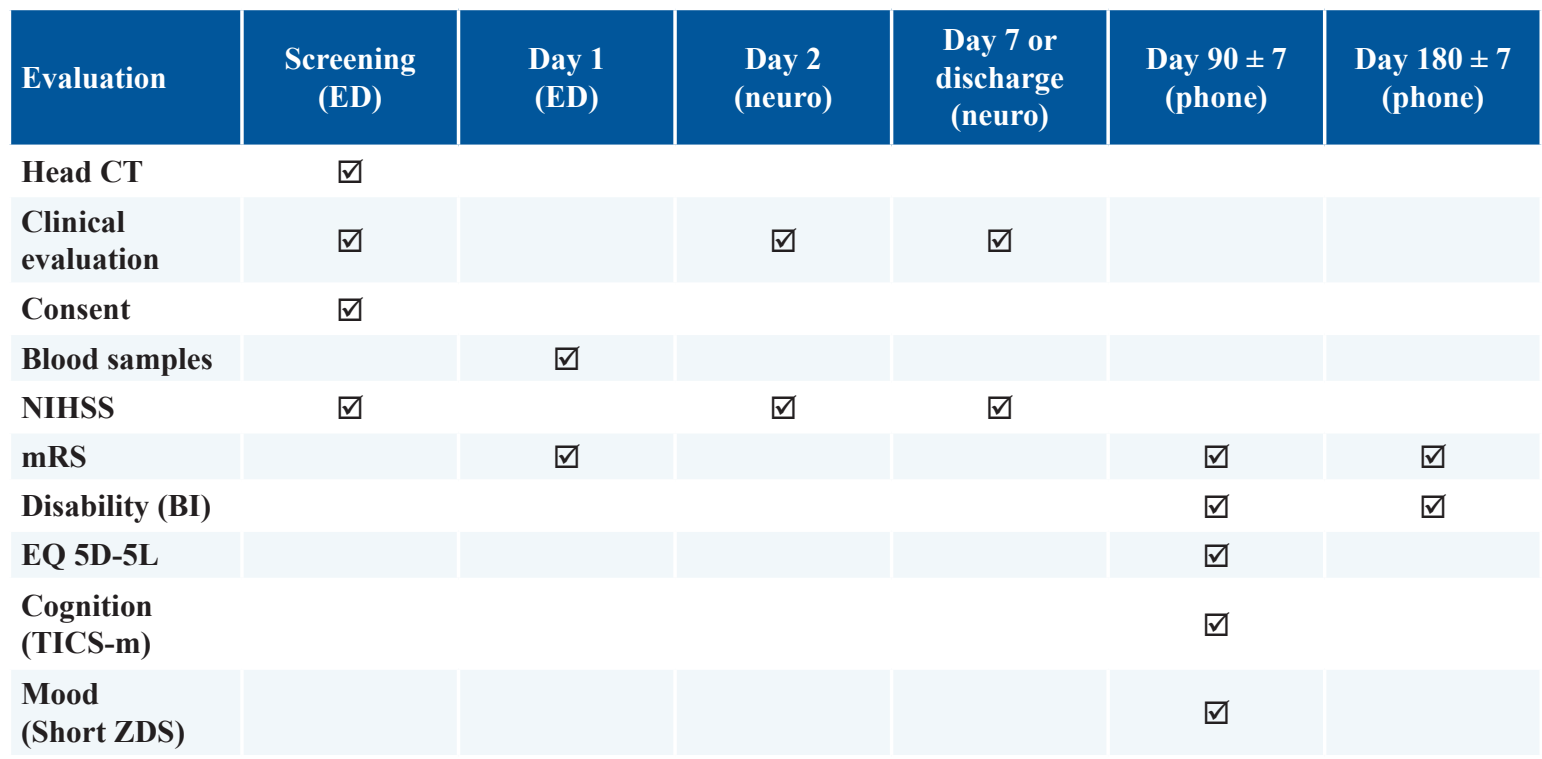

Legend: NIHSS - National Institute of Health Stroke Scale; mRS - modified Rankin Scale; BI - Barthel Index; EQ 5D5L - EuroQoL - Quality of Life; TICS-m - Telephone Interview for Cognitive Status-modified; ZDS - Zung Self-Rating Depression Scale. 


\section{Participants}

The enrollment process included adult (age over 18) patients diagnosed with sICH within the first 8 hours from ictus, for whom contact details had been documented and informed consent was obtained. Exclusion criteria consisted of secondary causes of ICH (e.g., trauma, known arterio-venous malformations, aneurysms, hemorrhagic transformation of ischemic stroke, thrombosis, thrombolysis, tumors, infections), Glasgow Coma Scale (GCS) $<8$ points, known thromboembolic disease, history of coagulopathy, recent ischemic events (e.g., stroke, myocardial infarction, peripheral arterial disease within the last 12 months), history of seizures, severe pre-ICH disability ( $\mathrm{mRS} \geq 4$ ), current treatment with heparin, LMWH, GPIIb/IIIa antagonists or oral anticoagulants, pregnancy or breastfeeding, scheduled neurosurgical intervention in the next 24 hours, scheduled hemostatic treatment, enrolment in other studies within the last 30 days, or terminal disease.

\section{Point-of-care devices}

All POC determinations have been conducted on analyzers currently used in advanced care Romanian ED (operated by ED nurses): Swelab Alfa Plus hematology analyser, Fujifilm Dry-Chem NX500 whole blood biochemistry analyser, and PathFast ${ }^{\mathrm{TM}}$ Mitsubishi Kagaku Iatron, Inc. analyser (for cTnI, hs-CRP and D-dimer). Personnel processing blood samples was not blinded to participants' diagnosis.

For study POC biomarkers (cTnI, hs-CRP and D-dimer), a total of 5 determinations were sampled previously to the participants' study enrolment due to case management reasons and it has been decided to accept those values as relevant for future analysis.

\section{Imaging}

Initial and control CT scans were performed on a General Electric Optima 64 scanner (GE Healthcare, Boston, Massachusetts, USA). Non-enhanced head CT images were acquired in the caudo-cranial direction (from the skull base to the vertex) using the following parameters: matrix, 512x512; collimation, $20 \times 0.625$ $\mathrm{mm} ; 120 \mathrm{kV} ; 250$ effective mAs, using a dose reduction algorithm (ASIR) of $50 \%$; rotation time, $1 \mathrm{~s} /$ rotation tube, at table speed $15 \mathrm{~mm} /$ rotation, with a pitch of 0.56 . The images were reconstructed as axial, coronal and sagittal 3-mm-thick images; the $3 \mathrm{~mm}$ width images were reconstructed with $3 \mathrm{~mm}$ increment. The post processing analysis was performed on a General Electric AW Server 2.0 workstation. The hemorrhage volume was measured by using manual segmentation with the inclusion of the entire area where the lesion was seen.

Two independent radiologists assessed diagnostic and control scans, blinded to patients' outcome. Consensus results were used for following analysis.

\section{Statistical analysis}

Statistical analysis was performed with Statistica (StatSoft, USA, v. 8). Descriptive statistics are reported as the number, percentage and associated $95 \%$ confidence intervals (reported in squared brackets) using an exact formula similar to that presented by Jäntschi [41] were reported for qualitative data. The value of media, first to third quartile in round brackets, and range as minimum to maximum value in curly brackets were reported for quantitative data.

\section{Results}

During the study period, 77,134 patients were treated in the ED of County Emergency Hospital ClujNapoca, Romania. Three hundred and seventy-five subjects had suggestive clinical aspects and an imaging ICH diagnosis $(0.50 \%$ [ $0.45 \%$ to $0.54 \%])$. Sixty-four percent of imagistic ICH subjects suffered a sICH $[59 \%$ to $69 \%$ ].

Thirty-nine subjects were included in the study $(16 \%[12 \%$ to $22 \%])$ and thirty-five remained under observation ( $90 \%$ [77\% to $97 \%]$ ); one withdrew and three underwent neurosurgery within 24 hours from inclusion (see Figure 1). The inclusion rate was 2.16 patients/ month. No patient refused the ED enrollment, though the EP was used in 12 cases (34.3\%) and in another 21 (60\%) the legal representative's consent was initially obtained in the ED. On day 90, 1/35 participants was not available for the telephone follow-up, compared to $4 / 35$ participants on day 180.

ED admission and inclusion times are presented in figure 2. The median time from symptom onset to ED presentation was 128 minutes (IQR 96 to 239), with 21/35 patients having presented within the first 3 hours from ictus. All participants had a diagnostic CT scan performed in our ED, with a median time of 170 minutes (IQR 126 to 317 ) between symptom onset and CT scan acquisition. ICH imaging diagnosis was available in a median time of 25 minutes (IQR 17 to 62 ) from presentation. More than half of the participants $(18 / 35)$ underwent the imaging investigation in the first 30 minutes from their arrival, with 9 of them within the first 20 minutes. Regarding symptom onset to POC sampling time, the median values for routine and study specific biomarkers were $150 \mathrm{~min}$ (IQR 117 to 259) and 237 minutes (IQR 193 to 384 ), respectively. The median times from patients' ED presentation to $\mathrm{CBC}$ results and study sampling were 12 minutes (IQR 6.5 to 20) and 100 minutes (IQR 74 to 139), respectively. 21/35 participants had routine POC panel results available within 15 minutes from ED arrival. 
Total ED patients

12.12.2016 - 11.06.2018

77,134
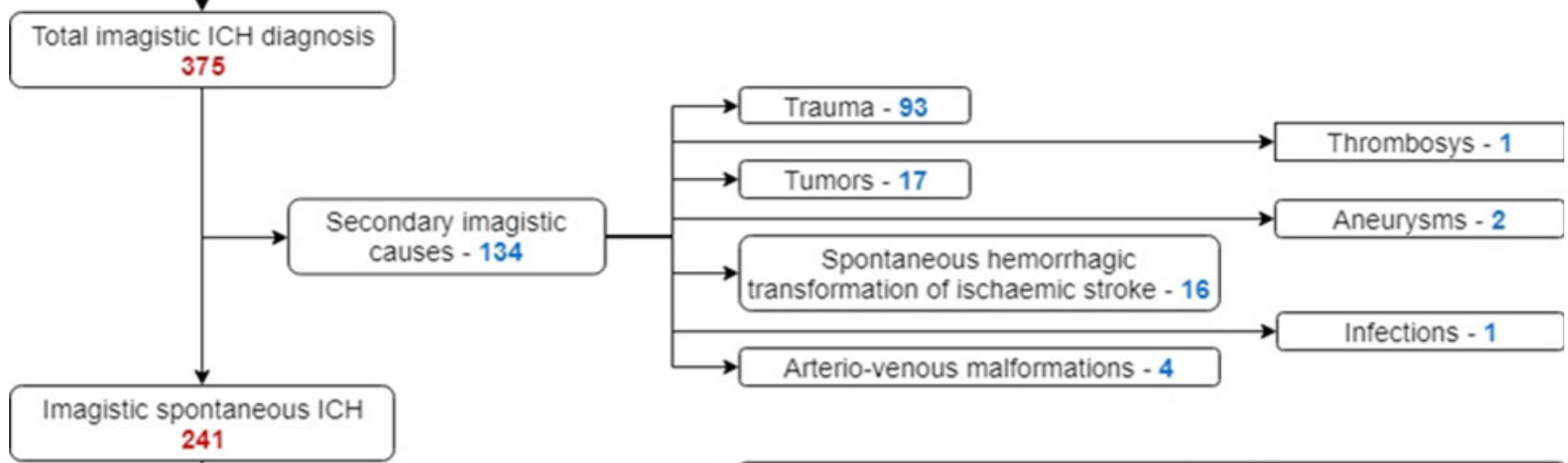

criteria - 151

(in various combinations)

\begin{tabular}{|c|c|}
\hline \multicolumn{2}{|c|}{ Late presentation - 74} \\
\hline \multicolumn{2}{|c|}{$\begin{array}{c}\text { GCS }<8 \text { points within } 8 \text { hours }-37 \\
\text { (total number of patients with GCS }<8-46 \text { ) }\end{array}$} \\
\hline & Known coagulopathy - 0 \\
\hline $\begin{array}{c}\text { Current use of anticoagulants, heparin, } \\
\text { LMWH, GPIIb/ IIla inhibitors - } 52\end{array}$ & \\
\hline \multirow{2}{*}{ Seizures - 16} & treatment - 0 \\
\hline & Recent PAD - 0 \\
\hline Known DVT - 7 & \\
\hline Recent ischemic stroke - 3 & Pregnancy - 0 \\
\hline Severe pre ICH disabi & $\begin{array}{c}\text { Enrolled in other ongoing } \\
\text { studies - } 0\end{array}$ \\
\hline Terminal disease - $\mathbf{2}$ & Planned neurosuraical \\
\hline Recent acute MI - 1 & intervention in the first \\
\hline No available patient information -4 & 24 hours -0 \\
\hline
\end{tabular}

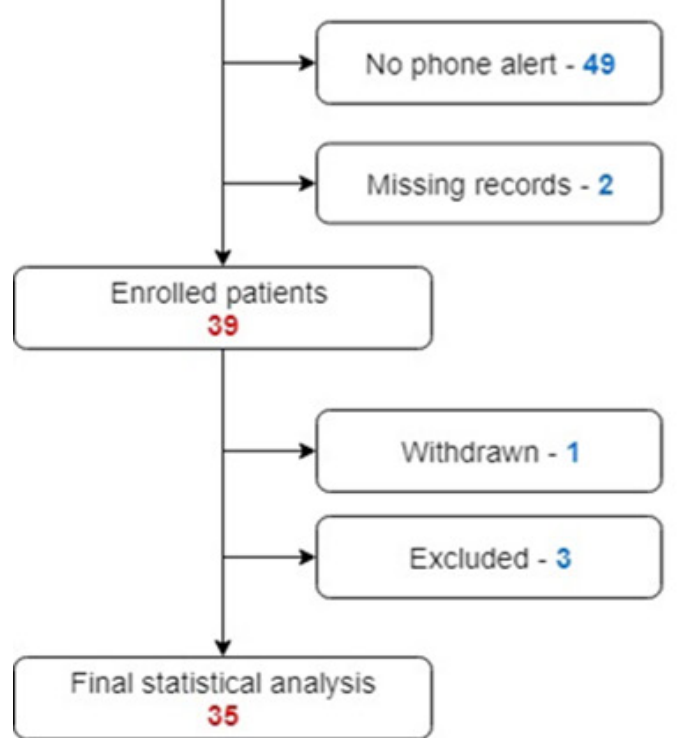

Figure 1. EsICH-bio STROBE diagram. 


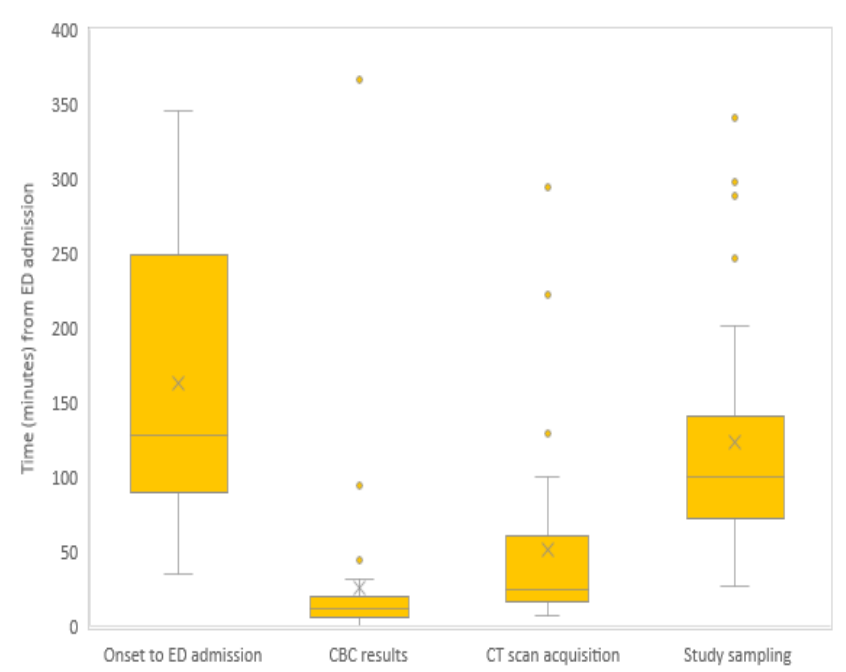

Figure 2. Spontaneous intracerebral hemorrhage admission and inclusion times within the emergency department (ED) (the $\times$ corresponds to the mean value, the horizontal line in the box to the value of median, the box to the $25^{\text {th }}$ and respectively $75^{\text {th }}$ percentile and the whiskers to the maximum and minimum values).

During the ED enrollment, no missing data were recorded on items incorporated into the objectives of the study (neurological assessment, study biomarkers and routine blood panel), yet interest is not constantly paid to certain cardiovascular risk factors such as alcohol consumption and smoking (missing data $22.8 \%$ and $11.4 \%$, respectively). Moreover, hematoma description is currently unstandardized, with items such as volume or 3-dimensions characterization being completely overlooked by the on-call radiologist. Fever was not documented on any participant, yet a high percentage of missing data on ED enrollment was observed on this item $(37 \%)$.

The demographics and past medical history of the investigated cohort are illustrated in table II. The participants had a mean age of 69.9 years (10.4 years standard deviation), with $6 / 35$ participants (17.1\%) older than 80 . Most frequently documented risk factors were hypertension, ischemic heart disease and diabetes mellitus. One-third of the hypertensive patients did not undergo any blood pressure (BP) lowering treatment.

Neurological status on admission was documented as GCS and NIHSS scores. The GCS values ranged from 8 to 15 , with 4 participants scoring a 10 or lower GCS. Upon enrolment, 4 participants had SBP values higher than $200 \mathrm{mmHg}$. During the first 24 hours from ictus, $30 / 35$ participants $(86 \%)$ required 3 or more BP lowering agents, with one patient requiring 10 pharmacological forms. The most frequently used BP lowering medication were enalapril, furosemide, and metoprolol, whilst calcium channel blockers were only used in 3 patients. No participant had a pre-stroke dependency greater than 2 on the mRS; 5 out of the 35 participants had a pre-stroke $\mathrm{mRS}$ of 1 . The clinical characteristics of the participants on ED admission are presented in table III.

Table II. Demographic data of study participants.

\begin{tabular}{|c|c|}
\hline & $\begin{array}{l}\text { Total cohort } \\
(n=35)\end{array}$ \\
\hline \multicolumn{2}{|l|}{ Demographics } \\
\hline $\begin{array}{l}\text { Age, years, median (IQR) } \\
>70 \text { years, } \mathrm{n}(\%)\end{array}$ & $\begin{array}{c}72(61.5-78) \\
18(51.4)\end{array}$ \\
\hline Male, n (\%) & $19(54.3)$ \\
\hline $\begin{array}{l}\text { Ethnic origin } \\
\text { Romanian, n (\%) } \\
\text { Hungarian, n (\%) }\end{array}$ & $\begin{array}{l}27(77.1) \\
8(22.9)\end{array}$ \\
\hline \multicolumn{2}{|l|}{ Medical history and general risk factors } \\
\hline Previous ischemic stroke or TIA, n (\%) & $8(22.9)$ \\
\hline Previous IHD, n (\%) & $11(31.4)$ \\
\hline Hypertension, n (\%) & $27(77.1)$ \\
\hline Diabetes mellitus, $\mathrm{n}(\%)$ & $10(28.6)$ \\
\hline $\begin{array}{l}\text { Smoking status } \\
\text { Former, } \mathrm{n}(\%) \\
\text { Active, } \mathrm{n}(\%) \\
\text { Never, } \mathrm{n}(\%)\end{array}$ & $\begin{aligned} 13 & (37.1) \\
2 & (5.7) \\
16 & (45.7)\end{aligned}$ \\
\hline $\begin{array}{l}\text { Alcohol intake } \\
\text { Past, n (\%) } \\
\text { Occasionally, n (\%) } \\
\text { Frequent/daily, n (\%) }\end{array}$ & $\begin{array}{c}2(5.7) \\
8(22.9) \\
10(28.6)\end{array}$ \\
\hline \multicolumn{2}{|l|}{ Medication history } \\
\hline Antiplatelet agent, n (\%) & $9(25.7)$ \\
\hline $\begin{array}{l}\text { Antihypertensive } \\
\text { Treatment = yes, n (\%) } \\
>2 \text { drugs, n }(\%)\end{array}$ & $\begin{array}{l}20(57.1) \\
10(28.6)\end{array}$ \\
\hline Lipid lowering agent, $\mathrm{n}(\%)$ & $10(28.6)$ \\
\hline
\end{tabular}

Legend: TIA: transient ischemic attack; IHD: ischemic heart disease.

Table III. Clinical characteristics of study participants on ED (study) admission.

\begin{tabular}{l|c} 
& $\begin{array}{c}\text { Total cohort } \\
(\mathbf{n}=\mathbf{3 5})\end{array}$ \\
\hline SBP $(\mathrm{mmHg})$, median (IQR) & $167(157-184)$ \\
$>170 \mathrm{mmHg}, \mathrm{n}(\%)$ & $17(48.6)$ \\
DBP $(\mathrm{mmHg})$, median $(\mathrm{IQR})$ & $89(79-101)$ \\
Heart rhythm & \\
Heart rate $(\mathrm{bpm})$ & $76(69-87.5)$ \\
Atrial fibrillation, n (\%) & $1(2.9)$ \\
NIHSS score, median (IQR) & $11(6.5-19)$ \\
$\geq 15, \mathrm{n}(\%)$ & $14(40.0)$ \\
GCS, median $(\mathrm{IQR})$ & $15(12.5-15)$ \\
Pre-stroke dependence (mRS), median $(\mathrm{IQR})$ & $0(0-0)$ \\
Temperature $\left({ }^{\circ} \mathrm{C}\right)$, median $(\mathrm{IQR})$ & $36.4(36.2-36.6)$
\end{tabular}

Legend: SBP: systolic blood pressure; DBP: diastolic blood pressure; bpm: beats per minute; NIHSS: National Institute of Health Stroke Scale; GCS: Glasgow Coma Scale; mRS: modified Rankin Scale. 
Paraclinical characteristics of the study cohort (Table IV) comprises CT scan aspects, and POC biomarkers values, both routine panel and study biomarkers. Hematoma location was most frequently supra-tentorial deep, with a median volume of $12.5 \mathrm{~cm}^{3}$ (IQR 7.26 to 25.52). Perihematoma edema (PHE) was present in all participants, with a median value of $9.00 \mathrm{~mm}$ (IQR 6.5 to 11.75).

Table IV. Baseline paraclinical characteristics of study participants on ED (study) admission.

\begin{tabular}{|c|c|}
\hline & $\begin{array}{l}\text { Total cohort } \\
(\mathrm{n}=\mathbf{3 5})\end{array}$ \\
\hline \multicolumn{2}{|l|}{ CT scan - Hematoma characteristics } \\
\hline $\begin{array}{l}\text { Hematoma location } \\
\text { Supra-tentorial Lobar, n (\%) } \\
\text { Supra-tentorial Deep, n (\%) } \\
\text { Infra-tentorial, n (\%) } \\
\text { Combination of above locations, n (\%) }\end{array}$ & $\begin{aligned} 6 & (17.1) \\
27 & (77.1) \\
1 & (2.9) \\
1 & (2.9)\end{aligned}$ \\
\hline $\begin{array}{l}\text { Hematoma volume }\left(\mathrm{cm}^{3}\right) \\
<30, \mathrm{n}(\%) \\
30-60, \mathrm{n}(\%) \\
>60, \mathrm{n}(\%)\end{array}$ & $\begin{array}{c}28(80.0) \\
4(11.4) \\
3(8.6)\end{array}$ \\
\hline IVH, n (\%) & $9(25.7)$ \\
\hline Midline shift > $10 \mathrm{~mm}, \mathrm{n}(\%)$ & $13(37.1)$ \\
\hline Mass effect, n (\%) & $29(82.9)$ \\
\hline Periventricular leucoaraiosis, n (\%) & $18(51.4)$ \\
\hline Lacunarism, n (\%) & $24(68.6)$ \\
\hline Cerebral atrophy, n (\%) & $20(57.1)$ \\
\hline $\begin{array}{l}\text { Compression of contralateral ventricle, } n \\
(\%)\end{array}$ & $28(80)$ \\
\hline Enlarged contralateral ventricle, n (\%) & $9(25.7)$ \\
\hline \multicolumn{2}{|l|}{ POC routine panel } \\
\hline WBC $\left(\times 10^{9} / \mathrm{L}\right)$, median $(\mathrm{IQR})$ & $9.30(6.7-11.3)$ \\
\hline GRA $\left(\times 10^{9} / \mathrm{L}\right)$, median $(\mathrm{IQR})$ & $5.9(4.1-8.3)$ \\
\hline LYM $\left(\times 10^{9} / \mathrm{L}\right)$, median $(\mathrm{IQR})$ & $1.9(1.4-2.4)$ \\
\hline MID $\left(\times 10^{9} / \mathrm{L}\right)$, median $(\mathrm{IQR})$ & $0.8(0.6-1.3)$ \\
\hline PLT $\left(\times 10^{9} / \mathrm{L}\right)$, median $(\mathrm{IQR})$ & $162(146-211)$ \\
\hline $\mathrm{Hb}(\mathrm{mg} / \mathrm{dL})$, median (IQR) & $13.60(12.7-14.85)$ \\
\hline Blood glucose (mg/dL), median (IQR) & $146(122-168)$ \\
\hline $\begin{array}{l}\text { Renal function } \\
\text { Creatinine }(\mu \mathrm{mol} / \mathrm{L}) \text {, median }(\mathrm{IQR}) \\
\text { BUN }(\mathrm{mg} / \mathrm{dL}), \text { median }(\mathrm{IQR})\end{array}$ & $\begin{array}{l}0.70(0.60-0.90) \\
17.5(13.8-21.4)\end{array}$ \\
\hline $\begin{array}{l}\text { Hepatic function } \\
\text { ASAT (U/L), median (IQR) } \\
\text { ALAT (U/L), median (IQR) }\end{array}$ & $\begin{array}{l}26.00(21.75-32.25) \\
23.00(14.00-31.00)\end{array}$ \\
\hline \multicolumn{2}{|l|}{ POC study biomarkers } \\
\hline hs-CRP (mg/L) median (IQR) & $2.49(0.679-4.17)$ \\
\hline cTnI (ng/mL) median (IQR) & $0.003(0.001-0.006)$ \\
\hline D-dimer ( $\mu \mathrm{g} / \mathrm{ml} \mathrm{FEU})$ median (IQR) & $0.91(0.665-2.82)$ \\
\hline
\end{tabular}

Legend: PHE: perihematoma edema; IVH: intraventricular hemorrhage; WBC: white blood cells; GRA: granulocytes; LYM: lymphocytes; MID: mid-cell fractions; PLT: platelets; $\mathrm{Hb}$ : hemoglobin; BUN: blood urea nitrogen; ASAT: aspartate aminotransferase; ALAT: alanine aminotransferase.

\section{Discussion}

Our results support the feasibility of ED-based enrolment in prospective research, with inclusion and follow-up rates resembling previously published ones $[3,42]$. Though sICH patients are diligently and promptly managed once admitted to $\mathrm{ED}$, late and irregular presentations in every third patient greatly limits enrolment opportunities. Nevertheless, ED POC testing provides a generous array of information on biological parameters, which theoretically could contribute to risk stratification and outcome improvement.

Thirty-nine patients were included over a period of 18 months, reaching similar inclusion and follow-up rates as other sICH reports [42]. If factoring in the 49 patients lost due to lack of study team activation, the inclusion rate could have doubled. Considering that none of the eligible patients refused ED enrollment and follow-up rates remained solid, we could speculate that ED is a favorable enrollment site for patients suffering of acute neurological conditions. Withal, certain aspects require immediate attention, if inclusion rate is to properly reflect the unique opportunity offered by an ED-based research enrollment.

The high proportion of no phone alert is of great concern. Apart from shortage of experienced EM researchers, a level one Romanian ED addresses all acute conditions, thus the workload is frequently considerable and prevents the on-call EM physician from dedicating time to patient screening and enrolment. As observed (figure 2), standard ED management for ICH patients is expeditious, yet the completion of the enrollment process and study sampling is prolonged (3.95 to 4.03 hours from stroke onset), even though still within previously published reports, ranging from 2 to 11.9 hours $[10,43,44]$. In contrast, items such as time from stroke onset to CT scan are lower in EsICH-bio cohort (3.48h vs. 6.9h) [45]. Subsequently, further efforts (e.g., training, academic and/ or professional recognition of both physicians and nurses) should be made as to increase the effective participation of EM professionals in prospective research.

The peculiarities of the Romanian emergency system and general medical education of the population are reflected by figure 2 . Late presentations $(30.7 \%$ of the potentially eligible cohort) probably mirror the population's lack of education on stroke recognition. Furthermore, EMS are regionally based, with all patients transferred to the nearest $\mathrm{ED}$, thus generating an additional delay due to the second ambulance transfer to a stroke centre. As of May 2018, a national stroke protocol [46] enables a pre-hospital and ED fast-track for each dispatch recognized stroke suspicion, aiming to shorten thrombolysis time.

Failure to document all required data when conducting an ED-based enrolment could be an expected side-effect of such a busy working environment. The current analysis emphasizes ED personnel's ability to prioritize the collection of indispensable study data, whilst 
overlooking items not customary used in their daily practice. Additionally, lack of standardized imaging interpretation potentially generates communication difficulties and even if of little benefit to research purposes, a uniformed diagnosis format would ensure better transparency in interdisciplinary collaboration. Electronic medical records could reduce frequency of missing data and empower evidence-based research, yet future studies are needed to quantify the overall impact of digitalization.

Educational efforts targeting EMS, ED physicians and the general population could shorten arrival times, enlarge enrollment ratio, and reduce missing data and interpretation errors, hence increasing prospects of a better outcome and contributing to a pertinent analysis of the Romanian sICH population. Networking and multi centre cooperation should be the focus of future initiatives, as access to a larger pool of critical patients would contribute to establishing pathways and risk scores.

As part of the secondary endpoint of the present study, demographical and POC biochemical data on ED enrolment were reported. The cohort's mean age was higher than previous data [3], with the preponderance of advanced age participants. Smoking and alcohol drinking are risk factors linked with the development of acute stroke [10], short-term mortality [45] and increase of hematoma volume [47]. Though of undisputed relevance for sICH long-term management, these risk factors are frequently overlooked in our ED, as little can be influenced during the hyperacute management, with alcohol drinking being more frequent than smoking in our cohort. The prevalence of ischemic heart disease, stroke (including transient ischemic attacks) in the EsICH-bio cohort was greater than previous reports [3]. Diabetes mellitus, a factor of resistant hypertension [16], is known to be associated with in-hospital mortality of hemorrhagic stroke [48] and was recorded in almost one third of our cohort, alongside median baseline glycemic values similar to a previous report [49]. Admission hyperglycemia is an independent risk factor for early mortality $[13,50]$, especially in nondiabetic patients [50].

Baseline ED vital signs included lower median values for both systolic and diastolic BP and HR [3,47,51]. Lack of compliance to BP lowering treatment was numerous among the cohort, despite being a known modifiable risk factor and predictor of mortality and outcome. Admission HR over $85 \mathrm{bpm}$ has been reported as being associated with increased mortality and worse mRS [16].

Routinely ED accessible POC biomarkers generate a comprehensive biological panel for any critical patient and we believe that sICH patients would benefit greatly if these data would be used for risk stratification and outcome prognostication. Inflammation seems to play a considerable part in sICH progression [7]. High WBC and neutrophils (NEU) admission values have been linked with admission hematoma volume and intraventricular hemorrhage (IVH),
END, death or major disability [10,11,44,50,52-54]. According to Kim et al. [45], a leukocytes value above $9,400 / \mathrm{mm}^{3}$ poses a 2.4 -fold increase risk of short-term mortality. Admission anemia has also been associated with larger ICH volumes [52], whilst admission lymphopenia is correlated with higher stroke severity, larger baseline hematoma volume and IVH, along with infection risk and 3-months mortality [7]. Nevertheless, our cohort's mean values are within accepted normal laboratory limits and not reaching published thresholds for prognostication utility. A similar conclusion can be extended to hs-CRP [10] and cardiovascular POC biomarkers, D-dimer [13] and cTnI [16].

However, certain new observations must be mentioned. Present results offer granulocytes as a surrogate for NEU and the question of POC granulocytes timerelated augmentation arises, if considering the one reported on NEU [53]. Monocytes are substituted by MID, which include eosinophils among others, whose contribution to sICH evolution is still uncharted [55]. Therefore, larger cohorts are required for validating the impact of POC testing in acute sICH.

Our study has some limitations. Firstly, and probably most important, is the single center location of this study. As very little data is available on the Romanian ED stroke population, multicenter research is needed to establish a detailed epidemiological profile, aiming to identify risk factors and probable complications. Secondly, the lack of resources for establishing a dedicated ED research team affected the inclusion ratio. As so, future prospective research should address this issue, if to take advantage of the large pool of eligible participants. Due to the particular setting of the hosting medical facility (ED, neurology and neurosurgery departments functioning in different locations), the degree of planned neurosurgical intervention could not be documented accurately, as 3 initially eligible patients worsened by the time they reached the neurosurgical ward and required immediate intervention (and were subsequently excluded from study). Lastly, 2 of the 241 potentially eligible patients' charts could not be accessed for the retrospective analysis due to administrative reasons.

\section{Conclusions}

Our study indicates that a prospective observational trial in sICH patients is feasible in the ED, with possible significant gains for improving urgent care of the critical pathologies currently stabilized within these departments. Reported inclusion and follow-up rates should encourage future ED-based prospective studies, in spite of prolonged arrival times leading to frequent late presentations. Current standard practices of on-paper medical records contribute to significant missing data.

Emergency department point-of-care devices can be easily accessible tools in the hyperacute management of sICH, a condition still seeking impactful strategies to reduce its economic and societal impact. 


\section{Acknowledgements}

Sorin Mihai Lăcan, Maria Petrescu, Mădălina Maria Pop, Alexandra Găvre, and Adina Gheorghiu formed the emergency department study team. Lavinia Manuela Lenghel and Csaba Csutak have reviewed the enrolment and follow-up CT scans.

\section{Supporting Agencies}

Iuliu Hatieganu University of Medicine and Pharmacy Cluj-Napoca, Romania, grant numbers PCD 7690/74/15.04.2016 and PCD 5200/64/01.03.2017.

\section{References}

1. Suter RE. Emergency medicine in the United States: a systemic review. World J Emerg Med. 2012;3:5-10.

2. Mendelow AD, Gregson BA, Rowan EN, Murray GD, Gholkar A, Mitchell PM, et al. Early surgery versus initial conservative treatment in patients with spontaneous supratentorial lobar intracerebral haematomas (STICH II): a randomised trial. Lancet. 2013;382:397-408.

3. Sprigg N, Flaherty K, Appleton JP, Al-Shahi Salman $\mathrm{R}$, Bereczki D, Beridze $\mathrm{M}$, et al. Tranexamic acid for hyperacute primary IntraCerebral Haemorrhage (TICH-2): an international randomised, placebo-controlled, phase 3 superiority trial. Lancet. 2018;391:2107-2115.

4. Ikram MA, Wieberdink RG, Koudstaal PJ. International epidemiology of intracerebral hemorrhage. Curr Atheroscler Rep. 2012;14:300-306.

5. Krishnamurthi RV, Ikeda T, Feigin VL. Global, Regional and Country-Specific Burden of Ischaemic Stroke, Intracerebral Haemorrhage and Subarachnoid Haemorrhage: A Systematic Analysis of the Global Burden of Disease Study 2017. Neuroepidemiology. 2020;54:171-179.

6. Fonseca S, Costa F, Seabra M, Dias R, Soares A, Dias C, et al. Systemic inflammation status at admission affects the outcome of intracerebral hemorrhage by increasing perihematomal edema but not the hematoma growth. Acta Neurol Belg. 2020 Jan 7, Ahead of print. doi: 10.1007/ s13760-019-01269-2..

7. Saand AR, Yu F, Chen J, Chou SH. Systemic inflammation in hemorrhagic strokes - A novel neurological sign and therapeutic target? J Cereb Blood Flow Metab. 2019;39:959-988.

8. Senn R, Elkind MS, Montaner J, Christ-Crain M, Katan M. Potential role of blood biomarkers in the management of nontraumatic intracerebral hemorrhage. Cerebrovasc Dis. 2014;38:395-409.

9. Kumar A, Kumar P, Misra S, Sagar R, Kathuria P, Vibha $\mathrm{D}$, et al. Biomarkers to enhance accuracy and precision of prediction of short-term and long-term outcome after spontaneous intracerebral haemorrhage: a study protocol for a prospective cohort study. BMC Neurol. 2015;15:136.

10. Di Napoli M, Parry-Jones AR, Smith CJ, Hopkins SJ, Slevin M, Masotti L, et al. C-reactive protein predicts hematoma growth in intracerebral hemorrhage. Stroke. 2014;45:5965.

11. Yu S, Arima H, Heeley E, Delcourt C, Krause M, Peng B, et al. White blood cell count and clinical outcomes after intracerebral hemorrhage: The INTERACT2 trial. J Neurol Sci. 2016;361:112-116.

12. Chiu CC, Li YN, Lin LJ, Hsiao CT, Hsiao KY, Chen IC. Serum D-dimer as a predictor of mortality in patients with acute spontaneous intracerebral hemorrhage. J Clin Neurosci. 2012;19:810-813.

13. Hu X, Fang Y, Ye F, Lin S, Li H, You C, et al. Effects of plasma D-dimer levels on early mortality and longterm functional outcome after spontaneous intracerebral hemorrhage. J Clin Neurosci. 2014;21:1364-1367.

14. Specogna AV, Turin TC, Patten SB, Hill MD. Factors associated with early deterioration after spontaneous intracerebral hemorrhage: a systematic review and metaanalysis. PLoS One. 2014;9:e96743.

15. Zhang L, Wang Z, Qi S. Cardiac Troponin Elevation and Outcome after Subarachnoid Hemorrhage: A Systematic Review and Meta-analysis. J Stroke Cerebrovasc Dis. 2015;24:2375-2384.

16. Lele A, Lakireddy V, Gorbachov S, Chaikittisilpa N, Krishnamoorthy V, Vavilala MS. A Narrative Review of Cardiovascular Abnormalities After Spontaneous Intracerebral Hemorrhage. J Neurosurg Anesthesiol. 2019;31:199-211.

17. Sandhu R, Aronow WS, Rajdev A, Sukhija R, Amin H, D'aquila K, et al. Relation of cardiac troponin I levels with in-hospital mortality in patients with ischemic stroke, intracerebral hemorrhage, and subarachnoid hemorrhage. Am J Cardiol. 2008;102:632-634.

18. Seblova J, Cimpoesu D, Khoury A, Revue E, Trenkler S. Prehospital emergency care systems in Europe - EuSEM prehospital section survey 2016. Eur J Emerg Med. 2018;25:446-447.

19. Falup-Pecurariu OG, Diez-Domingo J, Esposito S, Finn A, Rodrigues F, Spoulou V, et al. Clinical and laboratory features of children with community-acquired pneumonia are associated with distinct radiographic presentations. Eur J Pediatr. 2018;177:1111-1120.

20. Bouchiat C, Curtis S, Spiliopoulou I, Bes M, Cocuzza C, Codita I, et al. MRSA infections among patients in the emergency department: a European multicentre study. J Antimicrob Chemother. 2017;72:372-375.

21. Gräsner JT, Lefering R, Koster RW, Masterson S, Böttiger BW, Herlitz J, et al. EuReCa ONE-27 Nations, ONE Europe, ONE Registry: A prospective one month analysis of out-of-hospital cardiac arrest outcomes in 27 countries in Europe. Resuscitation. 2016;105:188-195.

22. Ferre RM, Chioncel O, Pang PS, Lang RM, Gheorghiade M, Collins SP. Acute heart failure: the role of focused emergency cardiopulmonary ultrasound in identification and early management. Eur J Heart Fail. 2015;17:12231227.

23. Nikolaou N, Castrén M, Monsieurs KG, Cimpoesu D, Georgiou M, Raffay V, et al. Time delays to reach dispatch 
centres in different regions in Europe. Are we losing the window of opportunity? - The EUROCALL study. Resuscitation. 2017;111:8-13.

24. Laribi S, Keijzers G, van Meer O, Klim S, Motiejunaite J, Kuan WS, et al. Epidemiology of patients presenting with dyspnea to emergency departments in Europe and the AsiaPacific region. Eur J Emerg Med. 2019;26:345-349.

25. Craig S, Kuan WS, Kelly A, Van Meer O, Motiejunaite J, Keijzers G, et al. Treatment and outcome of adult patients with acute asthma in emergency departments in Australasia, South East Asia and Europe: Are guidelines followed? AANZDEM/EuroDEM study. Emerg Med Australas. 2019;31:756-762.

26. Miró Ò, García Sarasola A, Fuenzalida C, Calderón S, Jacob J, Aguirre A, et al. Departments involved during the first episode of acute heart failure and subsequent emergency department revisits and rehospitalisations: an outlook through the NOVICA cohort. Eur J Heart Fail. 2019;21:1231-1244.

27. Georgescu V, Tudorache O, Nicolau M, Strambu V. Study regarding the survival of patients suffering a traumatic cardiac arrest. J Med Life. 2015;8 Spec Issue:103-9.

28. Tudorache O, Armean S, Georgescu V. An Utstein style based on a reporting system of the emergency department's cardiopulmonary resuscitation in an emergency hospital in Romania. J Med Life. 2013;6:446-450.

29. Tat RM, Golea A, Vesa ŞC, Ionescu D. Resistin-Can it be a new early marker for prognosis in patients who survive after a cardiac arrest? A pilot study. PLos One. 2019; 14:e0210666.

30. Tat RM, Golea A, Rahaian R, Vesa ŞC, Ionescu D. Resistin and Cardiac Arrest-A Prospective Study. J Clin Med. 2019;9:57.

31. Bădilă E, Negrea C, Rîpă A, Weiss E, Bartoş D, Tîrziu C. The Etiology of Syncope in an Emergency Hospital. Rom J Intern Med. 2016;54:173-178.

32. Pop C, Ianos R, Matei C, Mercea D, Todea B, Dicu D, et al. Prospective Study of Pulmonary Embolism Presenting as Syncope. Am J Ther. 2019;26:e301-e307.

33. Stoica A, Şorodoc V, Lionte C, Jaba IM, Costache I, Anisie E, et al. Acute cardiac dyspnea in the emergency department: diagnostic value of $\mathrm{N}$-terminal prohormone of brain natriuretic peptide and galectin-3. J Int Med Res. 2019;47:159-172.

34. Lionte C, Sorodoc V, Tuchilus C, Cimpoiesu D, Jaba E. Biomarkers, lactate, and clinical scores as outcome predictors in systemic poisons exposures. Hum Exp Toxicol. 2017;36:651-662.

35. Lionte C, Sorodoc V, Jaba E, Botezat A. Development and validation of a risk-prediction nomogram for inhospital mortality in adults poisoned with drugs and nonpharmaceutical agents: An observational study. Medicine (Baltimore). 2017;96:e6404.

36. Lionte C, Sorodoc V, Bologa C, Tuchilus C, Jaba E. Usefulness of Transthoracic Echocardiography Parameters and Brain Natriuretic Peptide as Mortality Predictors in Hospitalized Acutely Poisoned Patients: A Prospective
Observational Study. Basic Clin Pharmacol Toxicol. 2017;120:498-504.

37. Rus Ma D, Peek-Asa C, Baragan EA, Chereches RM, Mocean F. Epidemiology of Road Traffic Injuries Treated in a Large Romanian Emergency Department in Tîrgu-Mureş Between 2009 and 2010. Traffic Inj Prev. 2015;16:835-841.

38. Matei D, Groza I, Furnea B, Puie L, Levi C, Chiru A, et al. Predictors of variceal or nonvariceal source of upper gastrointestinal bleeding. An etiology predictive score established and validated in a tertiary referral center. J Gastrointest Liver Dis. 2013;22:379-384.

39. Dicu D, Pop F, Ionescu D, Dicu T. Comparison of risk scoring systems in predicting clinical outcome at upper gastrointestinal bleeding patients in an emergency unit. Am J Emerg Med. 2013;31:94-99.

40. Recensământul populației și locuințelor 2002 - Populatia dupa domiciliu si locul nasterii - Regiuni, Judete si Medii. Institutul Naţional de Statistică [Population and dwelling censor 2012]. 2019. Available from: https://insse.ro/cms/ files/RPL2002INS/vol1/tabele/t06.pdf.

41. Jäntschi L, Bolboacă SD. Exact probabilities and confidence limits for binomial samples: applied to the difference between two proportions. Sci WorldJournal. 2010;10:865878.

42. Sprigg N, Renton CJ, Dineen RA, Kwong Y, Bath PM. Tranexamic acid for spontaneous intracerebral hemorrhage: a randomized controlled pilot trial (ISRCTN50867461). J Stroke Cerebrovasc Dis. 2014;23:1312-1318.

43. Walsh KB, Campos B, Hart K, Thakar C, Adeoye O. M2 Monocyte Microparticles Are Increased in Intracerebral Hemorrhage. J Stroke Cerebrovasc Dis. 2017;26:23692375 .

44. Tao C, Hu X, Wang J, Ma J, Li H, You C. Admission neutrophil count and neutrophil to lymphocyte ratio predict 90-day outcome in intracerebral hemorrhage. Biomark Med. 2017;11:33-42.

45. Kim Y, Han MH, Kim CH, Kim JM, Cheong JH, Ryu JI. Increased Short-Term Mortality in Patients with Spontaneous Intracerebral Hemorrhage and its Association with Admission Glucose Levels and Leukocytosis. World Neurosurg. 2017;98:503-511.

46. Ministerul Sănătății. Acțiunea prioritară privind tratamentul accidentelor vasculare cerebrale acute. Procedura operațională standard privind traseul pacientului și protocolul terapeutic [Ministry of Health: Priority action regarding the treatment of acute stroke. Standard operational procedure regarding the the patient referral and therapeutic protocol] 2018 . Available from: https://www. neurology.ro/protocoale-si-ghiduri-meniu-main/protocolavc.

47. Cho TG, Lee JC, Park SW, Chung C, Nam TK, Hwang SN. Relationship between systemic thrombogenic or thrombolytic indices and acute increase of spontaneous intracerebral hemorrhage. $\mathrm{J}$ Cerebrovasc Endovasc Neurosurg. 2014;16:159-165.

48. Snarska KK, Bachórzewska-Gajewska H, KapicaTopczewska K, Drozdowski W, Chorąży M, Kułakowska 
A, et al. Hyperglycemia and diabetes have different impacts on outcome of ischemic and hemorrhagic stroke. Arch Med Sci. 2017;13:100-108.

49. Lee SH, Kim BJ, Bae HJ, Lee JS, Lee J, Park BJ, et al. Effects of glucose level on early and long-term mortality after intracerebral haemorrhage: the Acute Brain Bleeding Analysis Study. Diabetologia. 2010;53:429-434.

50. Elhechmi YZ, Hassouna M, Chérif MA, Ben Kaddour R, Sedghiani I, Jerbi Z. Prognostic Value of Serum C-Reactive Protein in Spontaneous Intracerebral Hemorrhage: When Should We Take the Sample? J Stroke Cerebrovasc Dis. 2017;26:1007-1012.

51. Di Napoli M, Behrouz R, Topel CH, Misra V, Pomero F, Giraudo A, et al. Hypoalbuminemia, systemic inflammatory response syndrome, and functional outcome in intracerebral hemorrhage. J Crit Care. 2017;41:247-253.
52. Adeoye O, Walsh K, Woo JG, Haverbusch M, Moomaw CJ, Broderick JP, et al. Peripheral monocyte count is associated with case fatality after intracerebral hemorrhage. J Stroke Cerebrovasc Dis. 2014;23(2):e107-e111.

53. Tapia-Pérez JH, Karagianis D, Zilke R, Koufuglou V, Bondar I, Schneider T. Assessment of systemic cellular inflammatory response after spontaneous intracerebral hemorrhage. Clin Neurol Neurosurg. 2016;150:72-79.

54. Zhang F, Ren Y, Fu W, Yang Z, Wen D, Hu X, et al. Predictive Accuracy of Neutrophil-to-Lymphocyte Ratio on LongTerm Outcome in Patients with Spontaneous Intracerebral Hemorrhage. World Neurosurg. 2019;125:e651-e657.

55. Chen Q, Liu J, Xu H, He W, Li Y, Jiao L, et al. Association Between Eosinophilic Leukocyte Count and Hematoma Expansion in Acute Spontaneous Intracerebral Hemorrhage. Front Neurol. 2019;10:1164. 